\title{
In vitro Anticancer Activity of Paclitaxel Incorporated in Low-melting Solid Lipid Nanoparticles
}

\author{
Mi-Kyung Lee ${ }^{\dagger}$ and Jae-Heon Yang ${ }^{1}$ \\ Department of Pharmaceutical Engineering \\ ${ }^{1}$ School of Pharmacy, Woosuk University, Jeonbuk 565-701, Korea \\ (Received June 3, 2009 • Revised June 8, $2009 \cdot$ Accepted June 11, 2009)
}

\begin{abstract}
Triglyceride solid lipid with medium chain fatty acid, tricaprin (TC), was used as a core matrix of lipid nanoparticles (LN) to solubilize water-insoluble paclitaxel and enhance the stability of nanoparticles by immobilization of incorporated drug in the solid core during storage at low temperature. In the present study, TC-LN containing paclitaxel was prepared by hot melt homogenization method using TC as a core lipid and phospholipids as stabilizers. The particle size of TC-LN containing paclitaxel was less than $200 \mathrm{~nm}$ and its zeta potential was around $-40 \mathrm{mV}$. Calorimetric analysis showed TC core could be solidified by freezing and thawing in the manufacturing process in which the hot dispersion should be prepared at elevated temperature and subsequently cooled to obtain solid lipid nanoparticles. The melting transition of TC core was observed at $27.5^{\circ} \mathrm{C}$, which was lower than melting point of TC bulk. The particle size of TC-LN remained unchanged when kept at $4^{\circ} \mathrm{C}$. Paclitaxel containing TC-LN showed comparable anticancer activity to the Cremophore ELbased paclitaxel formulation against human ovarian (OVCAR-3) and breast (MCF-7) cancer cell lines. Thus, lipid nanoparticles with medium chain solid lipid may have a potential as alternative delivery system for parenteral administration of paclitaxel.
\end{abstract}

Key words - Tricaprin, medium chain solid lipid, lipid nanoparticles, paclitaxel

Paclitaxel has been prescribed worldwide to treat the most aggressive forms of ovarian, lung and breast cancer, as well as AIDS-related Kaposi's sarcoma. ${ }^{1)}$ However, the clinical application of paclitaxel has been hampered by its low solubility in water and many other pharmaceutical solvents acceptable for parenteral administration. The currently available version of paclitaxel is formulated in a vehicle composed of a 50:50 (v/ v) mixture of Cremophor EL and dehydrated alcohol. However, Cremophor EL has been reported to cause serious hypersensitivity reactions ${ }^{2}$ and microcrystalline precipitation of paclitaxel can occur over time in aqueous infusion solutions due to its poor solubility in aqueous media. ${ }^{2,3)}$ Therefore, a great deal of effort has been directed toward development of alternative paclitaxel formulations that do not require the use of Cremophor EL.

We considered triglyceride lipids as alternative matrix for solubilization of water-insoluble agent, paclitaxel. Vegetable oils have been long used in fat emulsion for intravenous calorie supply and also been chosen as matrix to solubilize various water-insoluble, but lipid-soluble drugs. Unfortunately, pacli-

†본 논문에 관한 문의는 이 저자에게로

Tel : 063)290-1423, E-mail : leemk@woosuk.ac.kr taxel is not soluble enough in vegetable oils to prepare pharmaceutically available formulation effectively delivering clinical dose. ${ }^{4)}$ It seems that paclitaxel is not only water-insoluble but also oil-insoluble. In this regard, triglyceride lipid with shorter fatty acid chains than those in vegetable oils was studied as matrix in which paclitaxel could be solubilized or dispersed. Triglycerides with mono-saturated fatty acid chains show higher melting points with increasing fatty acid chain length. Tricaprin (TC), 1,2,3-tridecanoyl-rac-glycerol with the melting point of $31 \sim 32^{\circ} \mathrm{C}$ becomes a solid at room temperature and a liquid form at body temperature. Due to the solid property of tricaprin at room temperature, drug incorporated in the tricaprin core of lipid nanoparticles could be immobilized at the temperature below its melting point. Furthermore, it subsequently could minimize the chances of destabilization of the system caused by protrusion of the drug into the stabilizing layers, as seen in parenteral fat emulsions consisted with vegetable oil core. ${ }^{5}$ ) Tricaprin core of lipid nanoparticles would melt in the body followed by turning into emulsions and releasing the drug in a diffusion controlled mode.

In the present study, paclitaxel-loaded lipid nanoparticles were prepared using tricaprin as a core and phospholipids as dispersing and stabilizing agents. Their physicochemical properties such as particle size, surface charge, melting behavior of 
tricaprin and physical stability upon storage were evaluated. In vitro anti-cancer activity of paclitaxel incorporated into TClipid nanoparticles (TC-LN) was also determined using human ovarian cancer cell line, OVCAR-3, and human breast cancer cell line, MCF-7.

\section{Experimental}

\section{Materials}

Tricaprin (TC) was purchased from Sigma Chemical Co. (St. Louis, MO, USA). Egg phosphatidylcholine (eggPC) and distearoyl phosphatidylethanolamine-N-poly (ethylene glycol) 2000 $\left(\mathrm{PEG}_{2000} \mathrm{PE}\right)$ were purchased from Avanti Polar Lipids Inc. (Alabaster, AL, USA). All other chemicals were reagent grade and used without further purification.

\section{Preparation of Lipid Nanoparticles}

TC-LNs were manufactured by melt homogenization method. $\left.{ }^{6}\right) \mathrm{TC}$, eggPC and $\mathrm{PEG}_{2000} \mathrm{PE}$ were weighed into pearshaped 10-mL glass tube followed by sonication for $1 \mathrm{hr}$ at $60^{\circ} \mathrm{C}$ in bath type sonicator (Branson ${ }^{\circledR}$ ultrasonic cleaner, 3210R-DTH, Branson Ultrasonics Corp., CT, USA). Water for injection preheated at $60^{\circ} \mathrm{C}$ was added and sonicated for more than 3 hours until the milky and homogeneous crude emulsions were obtained. These crude emulsions were homogenized for 10 cycles at $100 \mathrm{MPa}$ using a high pressure homogenizer (Emulsiflex ${ }^{\circledR}$ EF-B3, Avestin Inc., Canada). The hot fine emulsions obtained by high pressure homogenization were cooled by three different methods; incubation at room temperature or $4^{\circ} \mathrm{C}$, or by instantaneously freezing by dipping into liquid nitrogen followed by thawing in water bath at room temperature. The resulting dispersions were stored at $4^{\circ} \mathrm{C}$.

\section{Determination of Solubility of Paclitaxel in Various Lipid Matrix}

Tricaprin, trilaurin and trimyristin were melted at the temperature $10^{\circ} \mathrm{C}$ higher than melting point. Approximately $10 \mathrm{mg}$ of paclitaxel was added to $100 \mathrm{mg}$ of melted lipids or oils such as cholesteryl oleate and medium chain triglyceride (MCT) oil. The mixture was sonicated for 1 hour and filtered through $0.22 \mu \mathrm{m}$. The filtrate was then weighed and dissolved with methanol. The solubility of paclitaxel in the resulting solution was determined using HPLC method as follows. Twenty microliter of the sample solution was injected into the column of an HPLC system consisting of a Hitachi L6200 pump, L4200 UV-VIS detector, L7200 Autosampler and D2500 Chromato-Integrator (Hitachi, Japan). The utilized analytical column was a Capcell Pak UG120 (C18, $5 \mu \mathrm{m}, 4.6 \times 150 \mathrm{~mm}$;
Shiseido, Japan). The mobile phase consisted of acetonitrile and $2 \mathrm{mM}$ phosphoric acid mixed at a ratio of 55:45. The flow rate was set at $1.2 \mathrm{~mL} / \mathrm{min}$, and the eluent was monitored at an absorption wavelength of $227 \mathrm{~nm}$.

\section{Measurement of Particle Size and Zeta Potential}

The mean particle size of the TC-core lipid nanoparticles was determined by dynamic light scattering method using Submicron Particle Sizer (Nicomp 370, Particle Sizing Systems, Inc., CA, USA). The lipid nanoparticles were diluted with water for injection to give an intensity of $300 \mathrm{~Hz}$ as recommended by manufacturer. The zeta potential of TC-LN was measured using Zetasizer 3000 (Malvern, UK).

\section{Microcalorimetric Analysis}

The melting behavior of the TC core of lipid nanoparticles was analyzed by VP-DSC Micro-calorimeter (MicroCal Inc., MA). TC-LN was prepared using three different methods of cooling of the intermediate hot emulsion; Freezing in liquid nitrogen and thawing, cooling at $4^{\circ} \mathrm{C}$, or slow cooling at room temperature (RT). The TC-LNs were incubated at $4^{\circ} \mathrm{C}$ for a week before the microcalorimetric analysis.

\section{In vitro Anti-cancer Activity}

Human ovarian cancer cell lines, OVCAR-3 and human breast cancer cell line, MCF-7, were used for in vitro anti-cancer activity study. Ovarian cancer cell line was inoculated at a density of $10^{4}$ cells in $200 \mu \mathrm{L}$ medium per well in 96-well plate and incubated for $24 \mathrm{hr}$. Subsequently, the medium was replaced with drug containing medium and the plate was incubated under $5 \% \mathrm{CO}_{2}$ at $37^{\circ} \mathrm{C}$ for $24 \mathrm{hr}$. To avoid the interference of lipid nanoparticles in the MTT assay, the lipid nanoparticle-containing medium was removed. And then, $180 \mu \mathrm{L}$ fresh medium and $20 \mu \mathrm{L}$ MTT solution $(5 \mathrm{mg} / \mathrm{mL}$ in PBS) were added into wells. Aspirating the medium after $3 \mathrm{hr}$ of incubation under $5 \% \mathrm{CO}_{2}$ at $37^{\circ} \mathrm{C}$ terminated internalization of MTT. After lysing cells with DMSO, the optical density at $570 \mathrm{~nm}$ was determined by microplate spectrophotometer (SPECTRAmax ${ }^{\circledR}$ 340PC, Molecular Devices Corp., CA). The same procedure was applied to breast cancer cell line, MCF-7, except 48-hr drug treatment.

\section{Results and Discussion}

\section{Solubility of Paclitaxel in Various Lipid Matrix}

To improve the solubility of paclitaxel, it must be needed to choose the matrix which can solubilize the highest amount of paclitaxel. Paclitaxel was not soluble in oil with long chain 
Table I-Solubility of paclitaxel in various lipid matrix

\begin{tabular}{lc}
\hline \hline Lipids & Solubility $(\mathrm{mg} / \mathrm{g})$ \\
\hline Tricaprin & 40.3 \\
Trilaurin & 15.0 \\
Trimyristin & 0.2 \\
Cholesteryl oleate & 3.7 \\
MCT oil & 6.4 \\
\hline
\end{tabular}

fatty acid such as cholesteryl oleate (Table I). It has been reported to be not only water-insoluble, but also poorly soluble in soybean oil $(0.3 \mathrm{mg} / \mathrm{mL}){ }^{7)}$ Tricaprin showed much higher solubility than other triglycerides with longer fatty chains such as trilaurin and trimyristin. Although MCT oil has been widely used as a vehicle for lipid-soluble drugs, the solubility of paclitaxel in MCT oil was lower than that in tricaprin. Based on these findings, tricaprin was chosen as a lipid matrix for lipid nanoparticles to solubilize paclitaxel.

\section{Particle Size and Zeta Potential}

TC-LNs showed mean diameters around $100 \mathrm{~nm}$ regardless of incorporating paclitaxel (Table II). The drug containing TCLNs remained within the injectable range for intravenous administration and were similar in size or smaller that fat emulsion presently being used for parenteral nutrition $(\sim 200$ $400 \mathrm{~nm})^{8}{ }^{8}$ The zeta potentials were around $-40 \mathrm{mV}$ regardless of paclitaxel incorporation, suggesting that paclitaxel did not significantly change the zeta potential of the LNs. Zeta potential of commercial fat emulsion has been reported in the range of approximately -40 to $-60 \mathrm{mV}$. $^{8)}$ Some researchers suggested that zeta potentials $>30 \mathrm{mV}$, optimum $>60 \mathrm{mV}$, are required for a full electrostatic stabilization. ${ }^{8}$ In this regard, zeta potential of the TC-core nanoparticles seems to be sufficiently high to stabilize the dispersion.

The TC-LNs showed a negative surface charge, which can be attributed to the stabilizing eggPC, as previously shown in commercial fat parenteral emulsion. ${ }^{8,9)}$ It was reported that a higher amount of phospharidylcholine was required to achieve stable and nano-sized particle dispersion of solid lipid than that

Table II-Mean particle diameter and zeta potential of TC-core lipid nanoparticles

\begin{tabular}{ccc}
\hline \hline Composition (weight ratio) & $\begin{array}{c}\text { Mean diameter } \\
(\mathrm{nm})^{\mathrm{a}}\end{array}$ & $\begin{array}{c}\text { Zeta potential } \\
(\mathrm{mV})^{\mathrm{b}}\end{array}$ \\
\hline TC:eggPC:PEG ${ }_{2000} \mathrm{PE}: \mathrm{TX}$ & & \\
100:30:3:0 (no drug) & $113.11 \pm 19.85$ & -42.8 \\
100:30:3:6 (with drug) & $88.39 \pm 11.36$ & -42.5
\end{tabular}

${ }^{\mathrm{a}}$ Mean diameter $\pm \mathrm{SD}$ of 8 batches

${ }^{\mathrm{b}}$ The mean of the 10 measurements

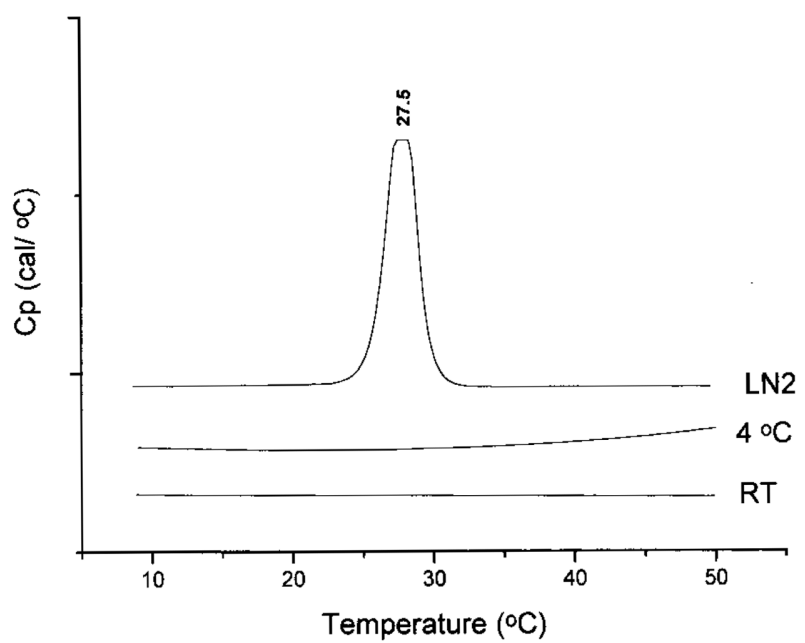

Figure 1-Microcalorimetric curve of TC-LN suspension when heated by $1^{\circ} \mathrm{C} / \mathrm{min}$ after 1 day incubation in a refrigerator. TC-LN was prepared by hot melt homogenization and subsequently cooled slowly at room temperature (RT), in $4^{\circ} \mathrm{C}$ chamber or by freezing in liquid nitrogen $\left(\mathrm{LN}_{2}\right)$ and then thawing.

used in parenteral fat emulsion consisting of liquid oil such as soybean oil. ${ }^{10)}$ Moreover, eggPC alone was not sufficient to stabilize the solid lipid nanoparticles; a pegylated phospholipids ( $\mathrm{PEG}_{2000} \mathrm{DSPE}$ ) was added to increase stabilization by steric hinderance. Therefore, TC-LNs were expected to be stable for a relatively long period.

\section{Melting Behavior of TC Core in Lipid Nanoparticles}

The properties of colloidal dispersed triglycerides differ from those of their bulk materials due to their colloidal size. This especially applies to the melting and crystallization behavior. ${ }^{11)}$ Therefore there could be possibility that TC-core in lipid nanoparticles was not solidified when cooled after hot homogenization in the manufacturing process. To confirm the solidification of the core, TC-LN was subjected to thermal analysis with heating the dispersion by $1^{\circ} \mathrm{C} / \mathrm{min}$ in VP-DSC Micro-calorimeter. As seen in Figure 1, TC-LN showed thermal transition peak only when cooled by freezing in liquid nitrogen and thawing. There were no melting peaks observed when cooled the hot homogenate at room temperature or in $4^{\circ} \mathrm{C}$ chamber suggesting that the TC core was not solidified. When frozen and thawed, TC-LN showed melting peak at $27.5^{\circ} \mathrm{C}$ which is significantly lower than the melting point of tricaprin bulk $\left(31 \sim 32^{\circ} \mathrm{C}\right)$. Based on the melting behavior of the TC core, it would be better to store TC-LN in a refrigerator for the stability of nanoparticles, especially LN with drug. In addition, TC core can be easily melted in the body and release the incorporated drug like emulsions. 


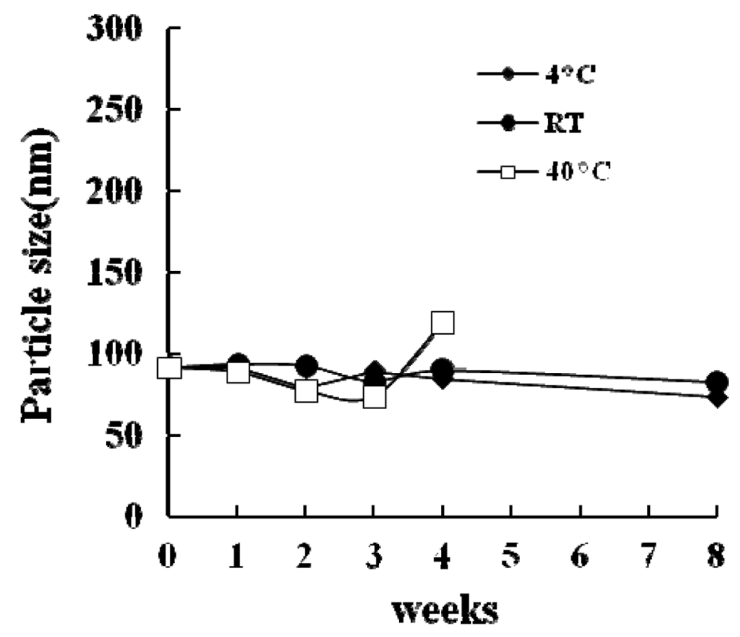

Figure 2-Physical stability of TC-LNs containing paclitaxel during storage at $4^{\circ} \mathrm{C}$, room temperature (RT) or $40^{\circ} \mathrm{C}$.

\section{Physical Stability of TC-LNs During Storage}

TC-LNs were stable at $4^{\circ} \mathrm{C}$ and RT for the observed period, 8 weeks, as shown in Figure 2. These results seem to be consistent with the findings of melting behavior of TC core and zeta potential suggesting that TC-LNs would be stable if stored at reduced temperature keeping the solid state of the core. In contrast, mean particle size slightly increased after 4 weeks at $40^{\circ} \mathrm{C}$, and finally phase separation occurred at 8 weeks. The instability at $40^{\circ} \mathrm{C}$ was likely due to liquefied state of the core.

\section{In vitro Anticancer Activity of Paclitaxel Incorporated} TC-LN

In human ovarian cancer cell line, OVCAR-3, paclitaxel in TC-LN was as effective as commercial formulation, Taxol ${ }^{\circledR}$ (Figure 3). Vehicles without paclitaxel were not cytotoxic up to the dose of vehicle containing $100 \mu \mathrm{M}$ paclitaxel (Figure 3).

In human breast cancer cell line, MCF-7, anticancer activities of paclitaxel in two formulations were similar (Figure 4). In contrast to ovarian cancer cells, TC-LN vehicle was more cytotoxic than Cremophor EL and ethanol mixture against MCF-7 at high concentrations (Figure 4). Although cytotoxicity induced by TC-LN containing paclitaxel was partially caused by LN itself at higher concentrations, survival rate was maintained $>90 \%$ up to $1 \mathrm{mM}$ paclitaxel incorporating dose. Thus, it was thought that the cytotoxic effect of TC-LN containing paclitaxel was mainly caused by paclitaxel rather than by vehicle. In MCF-7, 50\% and more cell killing by TC-LN containing paclitaxel was not observed when treated for $24 \mathrm{hr}$, but for $48 \mathrm{hr}$. Similar relationship between cytotoxicity and incubation time was reported for paclitaxel-containing pegylated liposomes. ${ }^{12)}$ Crosasso et al. exposed free drug and pacli-

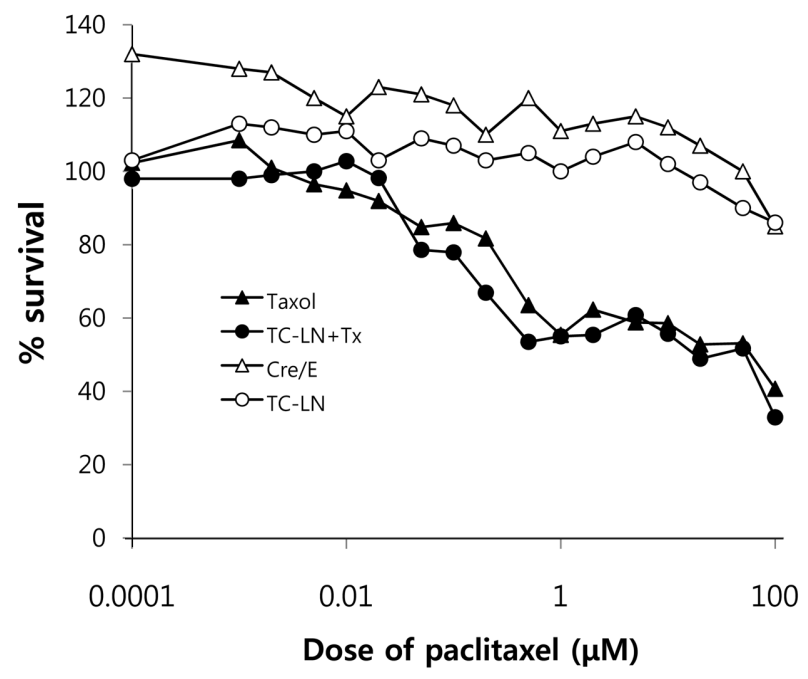

Figure 3-Cytotoxicity of paclitaxel in $\mathrm{TC}-\mathrm{LN}\left({ }^{\circ} \iota\right)$ or commercial formulation, $\operatorname{Taxol}^{\circledR}(\mathbf{O})$ in human ovarian cancer cell line, OVCAR-3, when treated for 24 hours. TC-LN without paclitaxel( $\mathrm{O})$ and a mixture of Cremophor ${ }^{\circledR}$ EL/ethanol (50:50) $(\triangle)$ were also shown for comparison. Each point represents mean of three independent experiments.

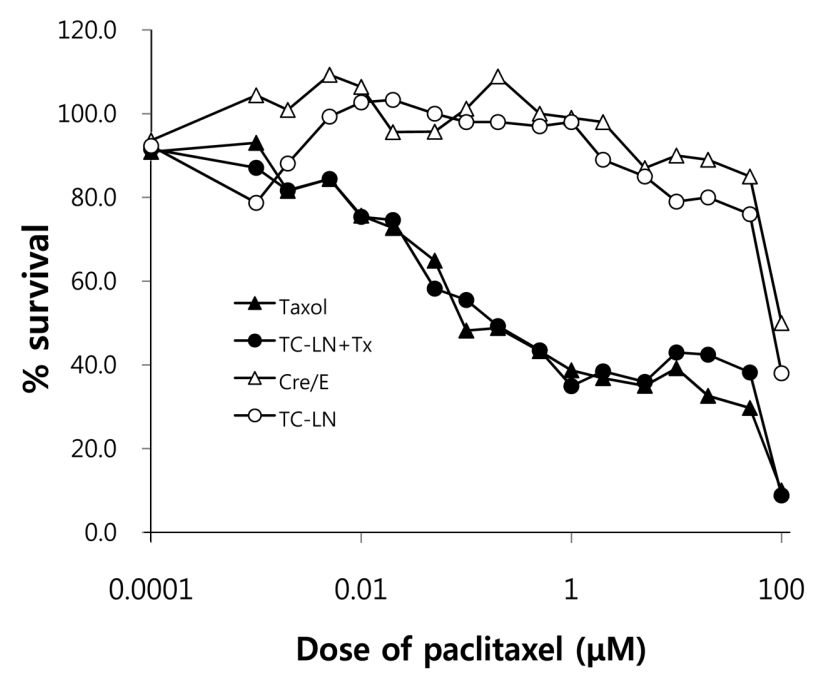

Figure 4-Cytotoxicity of paclitaxel in TC-LN( ) or commercial formulation, Taxol ${ }^{\circledR}(\mathbf{\Delta})$ in human breast cancer cell line, MCF-7, when treated for 48 hours. TC-LN without paclitaxel $(O)$ and a mixture of Cremophor ${ }^{\circledR}$ EL/ethanol (50:50) $(\triangle)$ were also shown for comparison. Each point represents mean of three independent experiments.

taxel-loaded liposomes for two different times ( $2 \mathrm{hr}$ and $48 \mathrm{hr}$ ). At both times, conventional and free drugs were nearly equipotent in human adenocarcinoma and human melanoma cell lines. Pegylated liposomes, on the contrary, were as active as the free drug only after $48 \mathrm{hr}$ of incubation. It was believed that the different behavior shown by the pegylated paclitaxel for- 
mulation compared to conventional liposomes might be due to the rigidifying effect of the presence of $\mathrm{PEG}_{5000}-\mathrm{DPPE}$ and cholesterol in the bilayer, which increased the time required for cell uptake and drug internalization. The similar phenomenon may have occurred in the present study. Paclitaxel should be released from LNs to present antiproliferative activity causing delayed anticancer activity compared to the commercial Cremophore EL-based formulation.

\section{Conclusion}

In the present work, paclitaxel was incorporated into lipid nanoparticles consisted of solid lipid with medium chain fatty acid, TC and phospholipids. The paclitaxel loaded TC-lipid nanoparticles were physically stable without any significant particle size change following long-term storage at $4^{\circ} \mathrm{C}$ and room temperature. Paclitaxel in TC-lipid nanoparticles showed anti-cancer activities against human ovarian and breast cancer cell lines comparable to those of commercially available Cremophore EL-based paclitxael formulation. These results suggest that TC-LN have promising potential as parenteral formulation for water-insoluble agents.

\section{Acknowledgements}

This work was supported by the Korea Research Foundation Grant funded by the Korean Government (KRF-2006-331E00440)

\section{References}

1) G.F. Ismael, D.D. Rosa, M.S. Mano and A. Awada, Novel cytotoxic drugs: old challenges, new solutions. Cancer Treat. Rev., 34(1), 81-91 (2008).
2) L.A. Trissel, Q.A. Xu and D.L. Gilbert, Compatibility and stability of paclitaxel combined with doxorubicin hydrochloride in infusion solutions. Ann. Pharemacother. 32, 1013-6 (1998).

3) Y. Zhang, Q.A. Xu, L.A. Trissel, D.L. Gilbert and J.F. Martinez, Compatibility and stability of paclitaxel combined with cisplatin and with carboplatin in infusion solutions. Ann. Pharmacother. 31, 1465-70 (1997).

4) H. Alan-Onjuksel, S. Ramakrishnan, H.B. Chai and J.M. Pezzuto, A mixed micellar formulation suitable for the parenteral administration of taxol. Pharm. Res. 11, 206-212 (1994).

5) J.S. Lucks, R.H. Müller and B. Konig, B. Solid lipid nanoaprticles (SLN) - an alternative parenteral drug carrier system. Eur. J. Pharm. Biopharm. 38, 33S (1992).

6) C. Schwartz, J.S. Mehnert, J.S. Lucks and R.H. Muller, Solid lipid nanoparticles (SLN) for controlled drug delivery. I. Production, characterization and sterilization. J. Control. Release. 30, 83-96 (1994).

7) B.D.Tarr, T.G. Sambandan and S.H. Yalkowsky, A new parenteral emulsion for the administration of taxol. Pharm. Res. 4, 162-165 (1987).

8) R.H. Müller and S. Heinemann, Fat emulsions for parenteral nutrition II: characterization and physical long-term stability of lipofundin MCT. Clin. Nutr. 11, 223-72 (1992).

9) C. Schwartz, J.S. Mehnert, J.S. Lucks and R.H. Müller, Solid lipid nanoparticles(SLN) for controlled drug delivery. I. production, characterization and sterilization. J. Control. Rel. 30, 83-96 (1994).

10) K. Westesen and T. Wehler, Physicochemical characterization of a model intravenous oil-in-water emulsion. J. Pharm. Sci. 82, 777-86 (1992).

11) K. Westesen and H. Bunjes, Do nanoparticles prepared from lipids solid at room temperature always possess a solid lipid matrix? Int. J. Pharm. 115, 129-131 (1995).

12) P. Crosasso, M. Ceruti, P. Brusa, S. Arpicco, F. Dosio and L. Cattel, Preparation, characterization and properties of sterically stabilized paclitaxel-containing liposomes. J. Control. Rel. 63, 19-30 (2000). 